\title{
The Differential Effects of Written and Spoken Presentation for the Modification of Interpretation and Judgmental Bias in Children
}

\author{
Stephanos P. Vassilopoulos \\ University of Patras, Greece \\ Simon E. Blackwell \\ MRC Cognition and Brain Sciences Unit, Cambridge, UK \\ Plousia Misailidi \\ University of Ioannina, Greece \\ Alexandra Kyritsi and Maria Ayfanti \\ University of Patras, Greece
}

\begin{abstract}
Background: Interpretation training programs, in which individuals are trained to interpret ambiguous scenarios in either a negative or benign way, have proven effective in altering anxiety-related cognitive biases in both children and adults. Aims: The current study investigated whether the effects of the interpretation training procedure in children are differentiated according to the mode of presentation of the training. Method: Ninety-four primary school children (aged 10-12 years) scoring above the mean on a social anxiety scale were randomly allocated to four groups, in which they were trained using written or spoken presentation of training materials in either the negative or benign direction. Results: For the negative training, children who heard the training material spoken aloud (spoken presentation) made more negative interpretations of ambiguous social events, compared to children who read the training material (written presentation). However, for the benign training, there was less clear evidence for a differentiation of the effects between the two modes of presentation, although children in the spoken presentation group performed better in a stressful task and showed a trend to rate their mood as more positive after the task than children in the written presentation group. Conclusions: These results not only forward our understanding of the
\end{abstract}

Reprint requests to Stephanos P. Vassilopoulos, Department of Primary Education, University of Patras, Patras 26 110, Greece. E-mail: stephanosv@upatras.gr

(C) British Association for Behavioural and Cognitive Psychotherapies 2013. The online version of this article is published within an Open Access environment subject to the conditions of the Creative Commons AttributionNonCommercial-ShareAlike licence <http://creativecommons.org/licenses/by-nc-sa/3.0/>. The written permission of Cambridge University Press must be obtained for commercial re-use. 
mechanism of the genesis of cognitive bias in children, but also highlight the need for further investigation of how to optimize the effectiveness of interpretation training in children.

Keywords: Cognitive bias modification, interpretation training, social anxiety, children.

\section{Introduction}

There is increasing evidence that childhood anxiety is associated with a tendency to disproportionately make threat interpretations of ambiguous information (for a review see Muris, 2010; Muris and Field, 2008). Moreover, over the past decade, experimental methods have been developed permitting interpretive bias to be induced in the laboratory. Referred to variously as bias training, bias induction or cognitive bias modification, these feedbacklearning paradigms are designed to directly modify cognitive biases in children and adults by introducing a contingency in which participants are reinforced for consistently resolving the meaning of an ambiguous vignette in either a negative or a benign way. Interpretive bias training procedures were initially designed to train negative interpretive biases in order to test causal hypotheses about the role of such biases in anxiety (Mathews and Mackintosh, 2000). Subsequently, numerous studies have demonstrated that biased interpretative processing is trainable and that it is causally implicated in anxiety vulnerability (see Beard, 2011, for a review). These procedures have also been adapted to train positive rather than negative interpretation (e.g. Holmes, Mathews, Dalgleish and Mackintosh, 2006), opening up the possibility of novel treatment development. Given that social anxiety in children has been consistently shown to be associated with negative interpretations (although it is less clear if these negative interpretations are distorted or a reflection of reality; see Miers, Blöte and Westenberg, 2011), recently the paradigms have been adapted for use with children (e.g. Vassilopoulos, Banerjee and Prantzalou, 2009). This may provide a particularly important avenue for research using a paradigm that can both elucidate causal mechanisms and has potential treatment implications: the former because it allows the opportunity to explore developmental aspects of disorders, and the latter because early intervention may be particularly valuable in reducing the incidence of disorders in later life.

One key area for investigation is how interpretation training paradigms may be made more effective in inducing the relevant bias. This can shed light both on potential factors affecting acquisition of negative biases in childhood, and also on how positive biases may be trained more effectively for therapeutic purposes. The current study aimed to investigate one specific factor that may be relevant in these circumstances: how the training material is presented.

Most of the scenario-based training procedures developed to modify cognitive biases in adults have either used visual presentation of written material (i.e. the text-based scenarios were read by participants themselves - Beard and Amir, 2008; Mathews and Mackintosh, 2000) or auditory presentation modality (i.e. participants listened to the scenarios through headphones - Murphy, Hirsch, Mathews, Smith and Clark, 2007; Holmes et al., 2006; Holmes, Lang and Shah, 2009). Only two adult studies so far have used an experimental design to directly compare the two presentation methods, and these reported no differential effect on induced interpretation bias or anxiety vulnerability between them (Mackintosh, Mathews, Yiend, Ridgeway and Cook, 2006; Standage, Ashwin and Fox, 2009). However, we cannot necessarily expect the same pattern of results found in adult studies to translate to children. The shift from visual to auditory presentation of training scenarios in adults was motivated 
by the idea that this would aid participants in imagining the scenarios, enhancing emotional processing of the training materials and thus the impact of the training itself (Holmes and Mathews, 2005). With children, the role of imagery instructions in the effectiveness of the training is an important area for further investigation (Vassilopoulos, Blackwell, Moberly and Karahaliou, 2012), but there are other reasons to suggest that having children listen to the training scenarios rather than reading them may enhance the impact of the training. First, children are less fluent readers than adults, so listening to the material may contribute to better encoding and further processing. For example, studies investigating the impact of presentation modality in working memory have consistently shown that when the modality of presentation is auditory rather than visual, superior recall for auditory items occurs, a finding known as the "modality effect" (e.g. Greene, Elliott and Smith, 1988). Moreover, there is preliminary evidence that when visual and auditory stimuli were presented simultaneously, children as young as 4-years old were more likely to process auditory stimuli than visual (Sloutsky and Napolitano, 2003), suggesting that children might have a preference for the auditory stimuli. Sloutsky and Napolitano (2003) speculated that this privileged processing of the auditory stimuli in the early years may have survival value as "it would be detrimental to a species' survival to receive all possible sensory information" (p.832). Thus, altering the modality of presentation of training material from visual (reading text) to auditory (listening to the material) may enhance the training effects.

Second, and perhaps more crucially, CBM-I, which uses verbal reinforcement-learning techniques (pairing a positive or negative outcome/explanation with an ambiguous event), is considered to closely mimic the social learning mechanisms by which cognitive biases are initially acquired (pairing a negative outcome/explanation with an ambiguous event, often through verbal report from parents - Lau, Molyneaux, Telman and Belli, 2011; Muris and Field, 2010). It may be that the ability to learn effectively via hearing verbal information, for example from parents, may have an important survival value in rapidly acquiring an understanding of what is or is not a source of danger, and such verbal information may play a particularly important role in fear acquisition in childhood (e.g. Field, 2006a; Field and Lester, 2010). Rachman (1977) has long stressed that children may become anxious and fearful when they hear that a situation is potentially dangerous, suggesting the verbal information transmission is one of the mechanisms through which fear and anxiety are transferred from parent to offspring. In support, there is substantial evidence that presenting information via the auditory modality can be highly effective for inducing or reducing fearrelated cognitive biases in children (e.g. Field, 2006a, b; Muris, Rassin, et al., 2009; Muris, Huijding, Mayer, van As and van Alem, 2011). Of course, in childhood any social learning that may lead to the shaping of cognitive biases via verbal information usually takes place in an interpersonal context - the verbal information is spoken, for example by a parent or other adult. Providing a similar interpersonal context for the CBM-I delivery - having the training materials spoken by an adult - may therefore not only capitalize on any advantages of delivering the information via the auditory modality, but also on any increased impact from the interpersonal context. There are therefore reasons to expect that the spoken presentation of the verbal training material by an adult may add to the effectiveness of an interpretation training program that involves text-based presentation of verbal information as used by Vassilopoulos et al. (2009). Thus, not only does this work have the potential to shed light on the potential mechanism of the genesis of interpretation bias but the findings may also carry important clinical implications. 
In the interpretation training procedure developed by Vassilopoulos and colleagues and used in the present study, children are presented with brief ambiguous scenarios describing various hypothetical social interactions that are age-appropriate. After reading an initial ambiguous scenario printed on a separate laminated card, children are required to choose between a benign and a negative explanation that follows, and they receive feedback on the correctness of the explanation. In order to assess the effect of the interpretation training procedure on children's spontaneous interpretation of novel ambiguous material, children complete a set of realistic ambiguous vignettes pre- and posttraining (Vassilopoulos et al., 2009,2012 ). The task is presented in a self-paced fashion and children read one vignette at a time and rate the extent to which each of the following interpretations would come to mind if they were in such a situation, providing an index of their interpretation bias.

Studies using the vignette method have demonstrated that it is possible to induce trainingcongruent interpretation biases in healthy and anxious children using experimental procedures (Lester, Field and Muris, 2011a, b; Muris, Huijding, Mayer and Hameetman, 2008; Muris, Huijding, et al., 2009; Vassilopoulos et al., 2009), thus mirroring the adult literature. Similar modification effects on interpretation bias have been reported for pubertal samples (Lothmann, Holmes, Chan and Lau, 2011; Salemink and Wiers, 2011). Further, selected socially anxious children showed a reduction in trait social anxiety after three sessions of benign interpretation training (Vassilopoulos et al., 2009), and children appear to be particularly vulnerable to acquiring interpretation biases about stimuli that are congruent with their normative fears for their age group (Lester et al., 2011a). Finally, Vassilopoulos et al. (2012) demonstrated that interpretation training in children can be effective with instructions to focus on the meaning of the vignettes alone and that modification congruent effects on children's self-imagery can be obtained with a minimal training consisting of 20 brief social scenarios (Vassilopoulos and Moberly, in press).

Previous research has also started to investigate the effects of interpretation training on children's emotional vulnerability. So far, three sessions of interpretation training have been found to significantly influence selected high socially anxious children's reported state anxiety about an anticipated upcoming social interaction (Vassilopoulos et al., 2009). Likewise, Lester et al. (2011a) found that children exposed to an animal-related behavioural avoidance task showed a trend for an increase in state anxiety in anticipation of performing this task after completing negative relative to positive bias modification. Nevertheless, Muris, Huijding, et al. (2009) failed to observe any training congruent effects on children's anticipated avoidance tendencies. These discrepant findings clearly mirror the contrasting results reported in the adult literature (see Beard, 2011; MacLeod and Mathews, 2012, for a review). Lester et al. (2011b) argued that a limitation of previous work with children is that none of these studies actually used a "live" stress task to assess children's actual behaviour and performance, and they set out to address this void by including a real ambiguous situation to measure children's behavioural avoidance. Nevertheless, they also failed to find evidence suggesting that the induced interpretation bias caused congruent changes in self-report state anxiety, although there was tentative evidence that change in interpretation bias was associated with avoidance behaviour (Lester et al., 2011b). Due to these inconsistent findings, another aim of the current study is to further investigate the effects of bias modification on emotional vulnerability by including a real stress-evoking task whereby participants have to complete a difficult anagram task designed to elicit a near 50\% completion success rate (Lester, Mathews, Davison, Burgess and Yiend, 2011). Past studies have demonstrated the effectiveness of the 
insoluble anagram task in elevating negative mood in participants (MacLeod, Rutherford, Campbell, Ebsworthy and Holker, 2002). Given that socially anxious individuals are sensitive to failure in performance situations (Rapee and Spence, 2004), this task seemed an appropriate "real life" test. We also sought to assess effects on objective and subjective performance because we reasoned that induced interpretation bias about an ambiguous hypothetical event would transfer to children's evaluation of their own performance during an ambiguous task.

In sum, the purpose of the present study was to examine whether written and spoken presentation of the training material differentially affect training-congruent change in interpretation bias and judgments of negative consequences in children (our primary outcome variables), and whether this translates into associated change in negative mood and emotional vulnerability. Based on previous work (e.g. Vassilopoulos et al., 2009, 2012), we anticipated that the interpretation training paradigm employed in this study would be successful in reducing negative interpretations but not in increasing benign interpretations, and that this effect would interact with mode of presentation. The study also aimed to examine this in relation to training both negative and benign interpretation, in order to inform our understanding of both the acquisition of negative biases and how best to remediate these.

\section{Method}

\section{Overview}

In an interpretation training paradigm, primary school children scoring above the mean on a self-report scale of social anxiety were randomly allocated to four groups, with two groups hearing the training material spoken by the experimenter resolved in either a negative $(n=$ $21)$ or a benign $(n=26)$ direction. The other two groups received the training material in a written form in either the negative $(n=20)$ or benign $(n=27)$ direction. We assessed children's social anxiety and depressive symptoms together with their initial interpretation and judgmental bias prior to training, and repeated most of these assessments after training one week later. Children also completed an anagram task - both before and after the training and reported their mood at various points during the experiment. Finally, participants were fully debriefed.

\section{Participants}

Participants were 94 primary school children enrolled in 5th and 6th grade classes from four public primary schools in the southwest of Greece, who were predominantly from a middle-class SES background. The participants were all Caucasian and ranged in age from $10-12$ years $(M=10.5, S D=0.6)$. This specific age group was selected because it has been found that preadolescents start showing an increased risk for developing social anxiety disorder (Miers, Blöte, de Rooij, Bokhorst and Westenberg, in press; Rapee and Spence, 2004). They were drawn from a population of 217 students who all completed the Social Anxiety Scale for Children-Revised (SASC-R) (La Greca and Stone, 1993). As previous work has demonstrated that more vulnerable individuals (e.g. anxious children) are more likely to respond to interpretation training (Muris et al., 2008), only children who scored above the 
mean score on SASC-R participated in the study in order to include children with medium to high levels of social anxiety. An equal number of boys and girls participated. We obtained verbal permission to perform the study from the principal of the school and each child's teacher. Parental consent was also obtained via letter and an opt-out procedure (only one parent refused). All children had normal or corrected-to-normal vision and hearing and were informed that they were free to withdraw or not participate in the study at any time without giving reason.

\section{Interpretation training program}

The procedure closely followed that of the training used in our previous studies (Vassilopoulos et al., 2009, 2012). Children were presented with 15 descriptions of hypothetical social events, written in the first person. The social descriptions and the following interpretations were identical for the benign and negative training conditions. Each of descriptions was presented in a form requiring participants to endorse one of the two alternative interpretations, one negative and one benign. Participants in the written condition read the descriptions, and participants in the spoken condition listened to the description being read out by the experimenter. After listening to/reading each event description printed on a separate card, participants answered a question designed to elicit the required response, by circling upon the card one of the two alternative interpretations that followed each description. An example of a training item is as follows:

During arts education, you ask your fellow student for one of his/her crayons, but he/she refuses.

What would you think if this happened to you?

(a) $\mathrm{He} /$ she dislikes me (negative interpretation)

(b) $\mathrm{He} / \mathrm{she}$ needs the crayon to finish his/her painting (benign interpretation)

After circling their response for each hypothetical event, participants in the written condition turned the card and saw the required response (negative or benign interpretation) printed on the back of each card and followed by "correct" feedback message written above it. Participants in the spoken condition only heard the "correct" response spelled out by the experimenter. Specifically, the experimenter said to participants as a group "the correct response is ..." followed by the negative or benign interpretation. No explanation for the "correct" response was provided.

\section{Measures}

Social anxiety assessment. Before training, social anxiety was measured with the Greek version of the Social Anxiety Scale for Children-Revised (SASC-R; La Greca and Stone, 1993). The SASC-R is a 22-item scale that assesses children's subjective feelings of social anxiety during various social situations and its correlates, including avoidance and inhibition. In the present study a 3 -point scale $(0=$ never true, $1=$ sometimes true, $2=$ always true $)$ was used instead of the original 5-point scale to make it more straightforward for the children. Cronbach's alpha was .55.

Depression assessment. Before training, depression was measured with the Greek version of the Children's Depression Inventory-Short Form (CDI; Kovacs, 1992). The CDI is a 
10-item questionnaire designed to assess the presence of depressive symptoms in children and adolescents aged between 7 and 17. The standard response scale $(1=$ absence of symptom, $2=$ mild symptom, 3 = definite symptom) was used. Cronbach's alpha was .66 . This measure was included for a better description of our sample.

Interpretation bias. A series of 18 ambiguous social scenarios (Vassilopoulos and Banerjee, 2008; Vassilopoulos et al., 2009) were presented, reflecting commonly occurring events that are relevant for the age group in question, such as inviting classmates to your birthday party some of whom do not reply, approaching a group of peers who stop talking upon seeing you, and going to your classmate's home to play together where nobody opens the door for you. Each description was followed by two thoughts that might sometimes occur to people in these situations. One interpretation always involved a negative judgment about oneself and the other interpretation involved a benign judgment of oneself or the situation. Participants rated the explanations in terms of the extent to which the explanations would be most likely to come to their mind if this event had happened to them, using a 5-point Likert-type scale ranging from 1 (I would not think of it at all) to 5 (I would think of it immediately). To assess judgmental bias (negative consequences), participants also answered the question: "How bad would it be for you if such an event had really happened?", using a 5-point Likert-type scale ranging from 1 (not at all bad) to 5 (very bad). Negative and benign interpretations for each situation were presented in a fixed random order. In order to control for unintentional order effects, half the event descriptions were presented at pre-assessment and the other half were presented at post-assessment in a counterbalanced order. Cronbach's alphas were $.72, .56, .80$ (for negative interpretations, benign interpretations, and emotional consequences, respectively) at pre-assessment, and $.86, .78, .89$ at post-assessment. Children's mean benign and negative interpretation bias scores were calculated for pre- and posttraining phase.

Visual analogue mood scales. Three visual analogue mood scales (VAMS) were administered to all participants at various points during the experimental procedure in order to assess their current mood state. In accordance with Lester et al. (2011a, b), the scales ranged from 0 (I do not feel anxious/depressed/happy at all) to 100 (I feel extremely anxious/depressed/happy). Instructions were either visually or auditorily presented, depending on the group assignment. The positive item was reversed-scored and a composite score was calculated so that higher scores indicate a higher negative mood state.

\section{Stressor task: anagram completion}

The task was adapted from the anagram stress task used by Lester, Mathews et al. (2011, Experiment 2). Two versions of the anagram task were developed and piloted for administration at baseline and test with difficulty level matched across versions. This task was piloted with six children to ensure that it was possible to solve approximately half of the anagrams within the allotted time ( 3 minutes). Each version consisted of five items varying in difficulty, determined by the number of letters (4-10) and extent of rearrangement (easy-small number of letters moved out of positions, e.g. school (loochs), hard-large number of letters moved out of position, e.g. unedsnt (student). In addition, one anagram in each version was age-inappropriate (e.g. verbalism) and another was impossible because it actually involved a 
pseudo word. Participants were instructed to solve as many anagrams as possible by writing down the correct words on a response sheet. Immediately afterward they completed two visual analogue scales (0-100) asking them to rate how they felt about their performance on the task (frustrated, successful). Actual performance was measured by the number of correctly solved anagrams.

\section{Debriefing}

All children who received negative interpretation training also completed a short benign interpretation training, consisting of five ambiguous social scenarios, each with two interpretations as before. Children were told to always try to choose the benign explanation for each situation and were given positive feedback when they did so.

\section{General procedure}

The study was conducted in two sessions; children were tested in small groups during both sessions. In the first session, the SASC-R, CDI, and the measures of interpretation and judgmental biases were administered. Participants then completed the first anagram task and the performance measures to establish baseline scores. All children were visited by the experimenter after approximately one week (mean interval $=7.42$ days, range 7-8 days). They were randomly assigned to either the spoken (they heard the stimuli and instructions spelled out by the experimenter) or the written presentation group (they read the instructions and stimuli, which were printed on cards), matched for age, gender, and social anxiety scores. At the beginning of the session participants rated their mood using a set of visual analogue scales. They were then introduced to the interpretation bias modification paradigm, which in the case of the written presentation group involved the participants working through the cards in self-paced fashion. In the case of spoken sequences, a warning "ready" was spoken 4 seconds before each new vignette, and 10 seconds were allowed for interpretation selection following each scenario. The experimenter was trained to read out the training material at a steady presentation rate, using as little emotional prosody as possible. The participants were asked, when reading/listening to the hypothetical events, to imagine themselves as the central character in the social situation. The interpretation training was then administered. For the spoken condition group the mean training time was 10 minutes whereas for the written condition group it was 15 minutes. During both training conditions, a second research assistant was present monitoring the procedure, but kept herself apart and intervened only to answer any questions regarding the hypothetical vignettes and the alternative interpretations or to interrupt any discussion between children. The re-administration of a second set of mood scales and the measures of interpretation and judgmental biases took place immediately after the interpretation training, using a new set of items for the interpretation and judgmental bias ratings, with sets used at test and re-test balanced over participants within groups. Finally, participants completed the second version of the anagram task, the mood (3rd set) and performance ratings and were debriefed. Specifically, children who received negative interpretation training were given the opportunity to complete a short benign interpretation training to eliminate any negative effects of the experiment (see Figure 1 for an outline of the experimental procedure). 


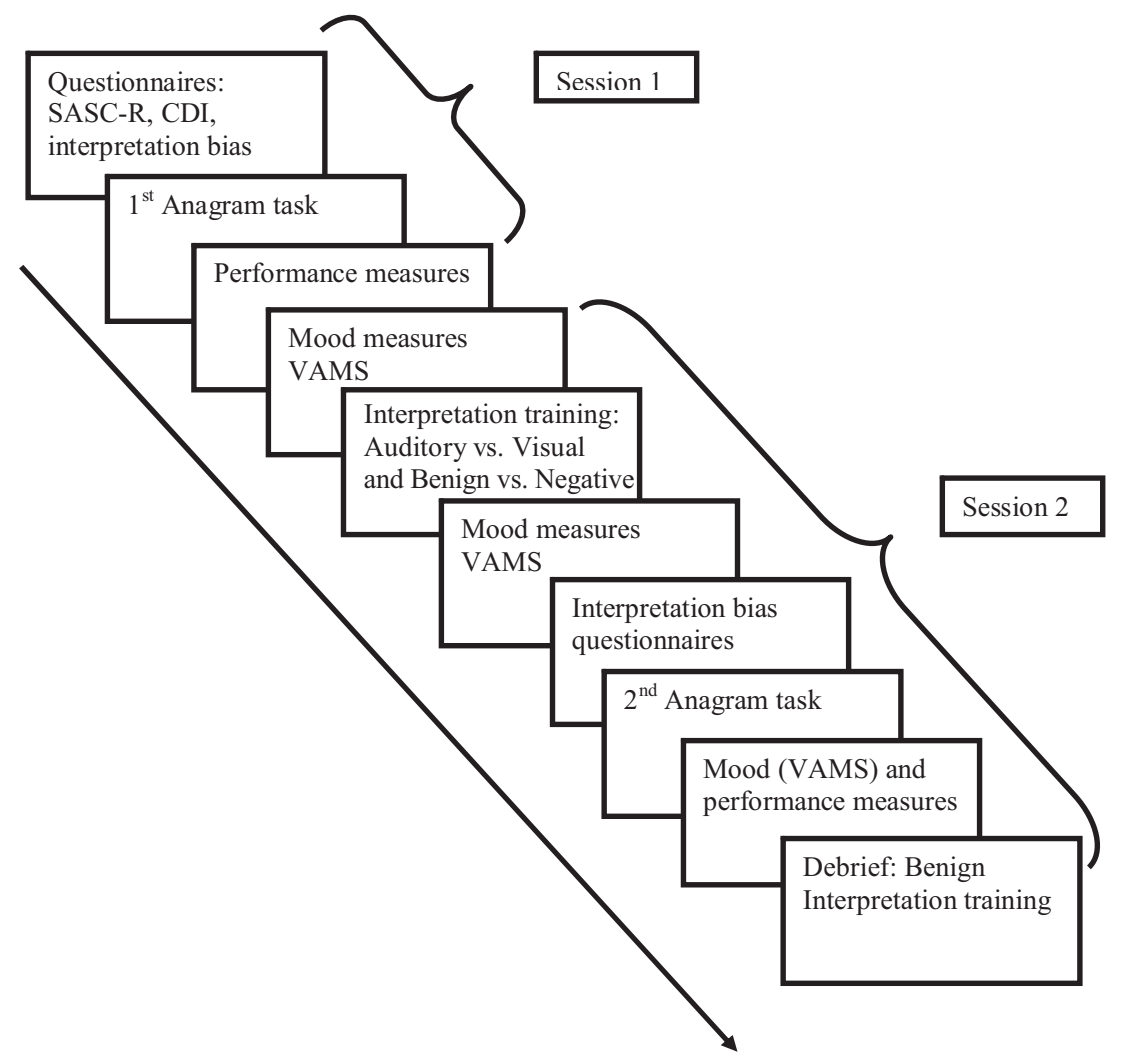

Figure 1. Outline of experimental procedure

\section{Results}

Table 1 displays means and standard deviations for all measures. All analyses were initially performed with sex of participants as a between-subjects' factor. There were no significant main effects or interactions with this variable; therefore, all analyses reported were collapsed across this variable. Separate three-way mixed-design Analyses of Variance were performed with interpretation training Condition (benign vs. negative) and Presentation (written vs. spoken) as the between subject factors and Time (pretraining vs. posttraining) as the withinsubject factor to test the hypotheses relevant to each dependent variable.

\section{Baseline psychological characteristics}

A Condition $\times$ Presentation ANOVA was conducted on all baseline measures of the dependent variables. There were no significant baseline differences in social anxiety, depression, current mood (visual analogue scales), negative interpretations, benign interpretations and emotional consequences between the written and spoken presentation factors in the negative and benign interpretation training groups. 
Table 1. Means (and standard deviations) of interpretation ratings, negative consequence ratings and trait measures for each condition on each occasion of testing

\begin{tabular}{|c|c|c|c|c|}
\hline & \multicolumn{2}{|c|}{ Benign } & \multicolumn{2}{|c|}{ Negative } \\
\hline & $\begin{array}{l}\text { Written } \\
(n=27)\end{array}$ & $\begin{array}{l}\text { Spoken } \\
(n=26)\end{array}$ & $\begin{array}{l}\text { Written } \\
(n=20)\end{array}$ & $\begin{array}{l}\text { Spoken } \\
(n=21)\end{array}$ \\
\hline \multicolumn{5}{|l|}{ Measure } \\
\hline Age & $10.44(.57)$ & $10.65(.93)$ & $10.65(.48)$ & $10.61(.49)$ \\
\hline Gender (female/male) & $(15: 12)$ & $(12: 14)$ & $(10: 10)$ & $(10: 11)$ \\
\hline Children's Depression Inventory & $12.22(2.06)$ & $13.11(2.47)$ & $12.75(2.24)$ & $12.47(2.97)$ \\
\hline Social Anxiety Scale for Children & $15.40(3.70)$ & $16.23(3.08)$ & $16.00(4.77)$ & $14.95(5.29)$ \\
\hline \multicolumn{5}{|l|}{ Negative interpretations: } \\
\hline Baseline & $3.04(.59)$ & $3.23(.86)$ & $3.18(.94)$ & $3.03(.83)$ \\
\hline Posttraining & $2.65(.88)$ & $2.85(1.19)$ & $2.51(.72)$ & $3.51(.94)$ \\
\hline \multicolumn{5}{|l|}{ Benign interpretations: } \\
\hline Baseline & $3.13(.55)$ & $3.02(.72)$ & $2.70(.74)$ & $2.98(.76)$ \\
\hline Posttraining & $3.42(.92)$ & $3.47(1.06)$ & $2.65(.74)$ & $2.89(.56)$ \\
\hline \multicolumn{5}{|l|}{ Negative consequences: } \\
\hline Baseline & $3.20(.76)$ & $3.53(.79)$ & $3.32(1.04)$ & $3.35(.76)$ \\
\hline Posttraining & $3.01(.86)$ & $3.15(1.26)$ & $2.92(1.02)$ & $3.62(.75)$ \\
\hline
\end{tabular}

\section{Interpretation bias}

For negative interpretations, there were significant main effects of Time, $F(1,90)=5.04, p=$ .03 , partial $\eta^{2}=0.05$, and Presentation, $F(1,90)=4.18, p=.04$, partial $\eta^{2}=0.04$, but no significant main effect of Condition, $F(1,90)<1$. There was no significant Condition $\mathrm{x}$ Time interaction, $F(1,90)=1.82, p=.18$, but there were significant interactions of Time $\mathrm{x}$ Presentation, $F(1,90)=7.39, p=.008$, partial $\eta^{2}=0.08$ and Condition $\times$ Presentation $\times$ Time, $F(1,90)=7.22, p<.009$, partial $\eta^{2}=0.07$. To examine this latter interaction, further Presentation $\times$ Time repeated measures ANOVAs investigated the effects of manipulations for the benign and negative training conditions separately. For the benign condition, there was a significant main effect of Time, $F(1,51)=6.62, p=.01$, partial $\eta^{2}=0.12$, no significant effect of Presentation, $F(1,51)<1$, and no significant Presentation $\mathrm{x}$ Time interaction, $F(1$, $51)<1$, indicating that in the benign condition children made fewer negative interpretations posttraining regardless of the mode of presentation of the training stimuli. For the negative condition, there was no significant main effect of Time, $F(1,39)<1$, a trend level effect of Presentation, $F(1,39)=3.48, p=.07$, partial $\eta^{2}=0.08$, and a significant Presentation $\times$ Time interaction, $F(1,39)=15.33, p<.001$, partial $\eta^{2}=0.28$. Post hoc analyses indicated that there was a significant increase in the number of negative interpretations of social events made by participants in the spoken presentation condition (hereafter "spoken") group, $t(20)=$ $2.05, p=.05$, whereas there was in fact a significant decrease in the number of negative interpretations made by participants in the written presentation condition (hereafter "written") group, $t(19)=3.82, p=.001$. Additionally, within the negative condition, although there was no significant difference between presentation conditions at pre-training, $t<1$, participants in the spoken group were more likely to endorse negative interpretations than did participants in the written group at posttraining, $t(39)=3.80, p<.001$ (see Figure 2). 


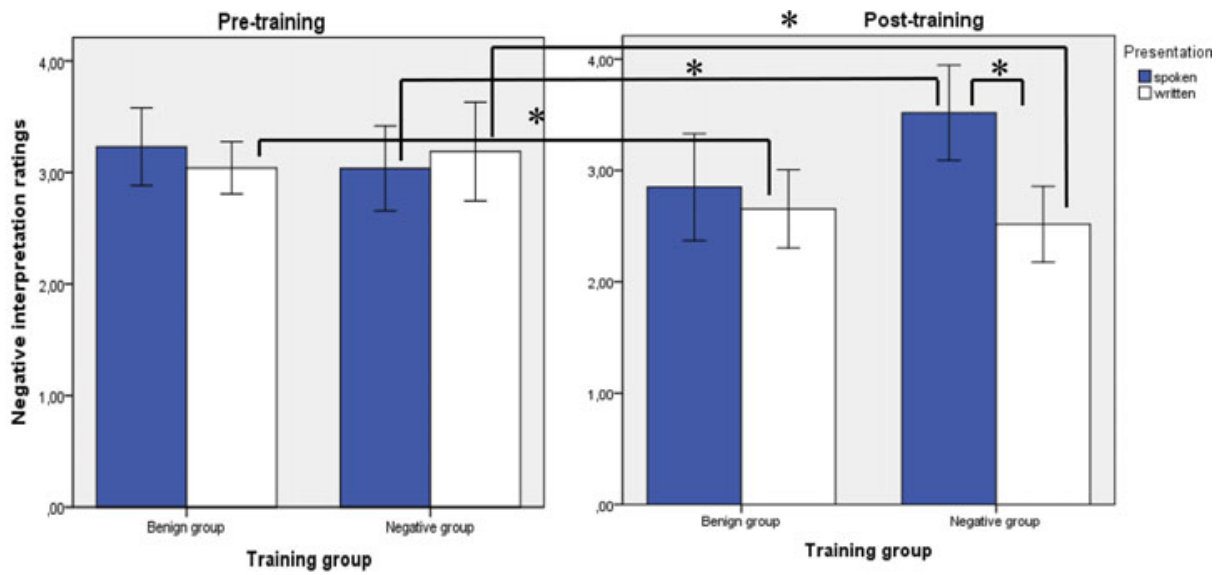

"Means differ significantly $(p \leq .05)$

Figure 2. (Colour online) Pre- and posttraining mean ratings for negative interpretations of ambiguous social events for each experimental condition (error bars represent $95 \%$ confidence intervals)

The analysis of benign interpretation ratings revealed a trend level main effect of Time, $F(1,90)=2.91, p=.09$, partial $\eta^{2}=0.03$, a significant main effect of Condition, $F(1,90)=$ 10.87, $p=.001$, partial $\eta^{2}=.11$, but no significant main effect of Presentation, $F(1,90)$ $<1$. There was a significant Condition $\mathrm{x}$ Time interaction, $F(1,90)=6.24, p=.01$, partial $\eta^{2}=.07$, but the Presentation $\mathrm{x}$ Time and Condition $\mathrm{x}$ Presentation $\mathrm{x}$ Time interactions were not significant, $F \mathrm{~s}<1$. Post hoc analyses showed that participants in the benign condition tended to make more benign interpretations over time, $t(52)=2.93, p=.005$, whereas for participants in the negative condition there was no significant pre-post difference, $t<1$ (see Table 1).

\section{Negative emotional consequence estimates}

Changes in estimates of negative emotional consequences of the hypothetical social events from pretraining to posttraining were examined using a similar mixed ANOVA. There were trend level main effects of Time, $F(1,90)=2.85, p=.10$, partial $\eta^{2}=0.03$, and Presentation, $F(1,90)=3.30, p=.07$, partial $\eta^{2}=0.04$, but no main effect of Condition, $F(1,90)<$ 1. The interactions were non-significant for Condition $\mathrm{x}$ Time, $F(1,90)=1.13, p=.29$ and Presentation $\mathrm{x}$ Time, $F(1,90)=1.39, p=.24$. However, there was again a significant interaction of Condition $\mathrm{x}$ Presentation $\mathrm{x}$ Time, $F(1,90)=4.14, p=.04$, partial $\eta^{2}=.04$. To decompose the interaction further Presentation $\times$ Time repeated measures ANOVAs were carried out for each training condition separately. For the benign condition there was only a near significant main effect of Time, $F(1,51)=3.43, p=.07$, partial $\eta^{2}=.06$, suggesting that participants tended to report less negative emotional consequences at posttraining than at pretraining. There were no significant effects of Presentation, $F(1,51)=1.29, p=.26$ or Presentation $\mathrm{x}$ Time, $F(1,51)<1$. However, for the negative training condition, while there were no significant main effects of Time, $F(1,39)<1$, or Presentation, $F(1,39)=1.93, p=$ .17 , the Presentation $\mathrm{x}$ Time interaction was found to be significant, $F(1,39)=7.02, p=.01$, 
partial $\eta^{2}=.15$. Post hoc analyses showed that for the written group there was a significant decrease in negative emotional consequence ratings over time, $t(19)=2.11, p=.04$, whereas for the spoken group there was a non-significant increase in negative consequence ratings, $t(20)=1.60, p=.12($ see Table 1$)$.

\section{Stressor task: anagram completion}

Changes in the number of anagrams correctly completed were tested using a similar mixed ANOVA. There were significant main effects of Time, $F(1,90)=7.86, p=.006$, partial $\eta^{2}=$ .08 , and Condition, $F(1,90)=10.61, p=.002$, partial $\eta^{2}=.11$, but not Presentation, $F<1$. There was a significant Condition $\mathrm{x}$ Time interaction, $F(1,90)=10.72, p=.002$, partial $\eta^{2}=$ .11 , and a trend level Presentation $\mathrm{x}$ Time interaction, $F(1,90)=3.40, p=.07$, partial $\eta^{2}=.04$, but no Condition $\mathrm{x}$ Presentation $\mathrm{x}$ Time interaction, $F(1,90)=2.05, p=.16$, partial $\eta^{2}=.02$. As previously, Presentation $\times$ Time repeated measures ANOVAs were carried out for each training condition separately. In the benign condition, there was no main effect of time, $F<1$, but a significant main effect of Presentation, $F(1,51)=5.78, p=.02$, partial $\eta^{2}=.10$, and a significant Presentation $\mathrm{x}$ Time interaction, $F(1,51)=5.49, p=.02$, partial $\eta^{2}=.10$. While children in both the spoken and written groups solved equal numbers of anagrams pretraining, $t(51)<1$, children in the spoken group solved significantly more anagrams than those in the written posttraining, $t(51)=3.59, p=.001$. In the negative condition, there was a significant main effect of time, $F(1,39)=19.38, p<.001$, but no main effect of Presentation, $F(1,39)=2.42, p=.13$, or interaction, $F<1$, indicating that regardless of mode of presentation of training materials, children in the negative condition solved fewer anagrams posttraining.

Participants' evaluation of performance (frustration, successfulness) was submitted to the same mixed ANOVA analysis. For successfulness ratings there was no significant main effect or interaction $(F \mathrm{~s}<1.5)$. For frustration ratings, there were no significant main effects of Time, $F(1,90)=2.03, p=.16$, or Presentation, $F<1$, but a trend level main effect of Condition, $F(1,90)=3.57, p=.06$, partial $\eta^{2}=.04$. The Condition $\mathrm{x}$ Time and Presentation $\mathrm{x}$ Time interactions were non-significant, $F<1$, but there was a significant interaction of Condition $\mathrm{x}$ Presentation $\mathrm{x}$ Time, $F(1,90)=4.34, p=.04$, partial $\eta^{2}=$ .05. To examine this interaction, further Presentation $\times$ Time repeated measures ANOVAs investigated the effects of manipulations for each training condition separately. For both benign and negative conditions no significant or trend level effects $(F \mathrm{~s} \leqslant 2.5)$ were found (see Table 2).

\section{Mood ratings}

Before running the analyses, the normality in the distribution of mood variables was assessed. No deviations from normal distributions were found (absolute values of skewness $<2$, absolute values of kurtosis $\leqslant 1$; Muthén and Kaplan, 1992). There were three mood measurements during the study: (1) immediately prior to training; (2) immediately after the training; and (3) after the $2^{\text {nd }}$ anagram task. They were organized by considering the first and second set of mood scales as part of the cognitive phase (interpretation training) and these scales measure how training directly affects mood. The third set of mood scales was considered as part of the stress phase of the study (insoluble anagram task) and change in 
Table 2. Mean (and standard deviations) ratings for the stressor task used in the study, and mood ratings

\begin{tabular}{|c|c|c|c|c|}
\hline & \multicolumn{2}{|c|}{ Benign } & \multicolumn{2}{|c|}{ Negative } \\
\hline & $\begin{array}{l}\text { Written } \\
(n=27)\end{array}$ & $\begin{array}{l}\text { Spoken } \\
(n=26)\end{array}$ & $\begin{array}{l}\text { Written } \\
(n=20)\end{array}$ & $\begin{array}{l}\text { Spoken } \\
(n=21)\end{array}$ \\
\hline \multicolumn{5}{|c|}{ Anagram Task: Actual performance (number of correct responses (/5) } \\
\hline Baseline & $2.18(.73)$ & $2.23(.76)$ & $2.30(.92)$ & $2.00(.63)$ \\
\hline Test & $1.92(.47)$ & $2.57(.80)$ & $1.70(.57)$ & $1.47(.51)$ \\
\hline \multicolumn{5}{|c|}{ Anagram Task: Performance evaluation $(0-100)$} \\
\hline \multicolumn{5}{|c|}{ Successfulness: } \\
\hline Baseline & $69.25(25.10)$ & $79.23(19.58)$ & $62.00(33.81)$ & $68.57(33.20)$ \\
\hline Test & $72.59(30.33)$ & $68.07(30.59)$ & $64.0(41.59)$ & $69.52(29.23)$ \\
\hline \multicolumn{5}{|l|}{ Frustration: } \\
\hline Baseline & $29.25(32.09)$ & $21.53(29.21)$ & $33.50(35.72)$ & $45.71(42.02)$ \\
\hline Test & $18.51(23.64)$ & $28.07(30.33)$ & $33.00(41.68)$ & $24.28(32.18)$ \\
\hline \multicolumn{5}{|c|}{ Mood ratings $(0-100)$ : } \\
\hline Time 1 & $15.06(15.28)$ & $11.15(14.60)$ & $20.66(16.87)$ & $17.77(22.95)$ \\
\hline Time 2 & $16.30(18.10)$ & $12.30(14.07)$ & $18.83(16.01)$ & $16.03(20.93)$ \\
\hline Time 3 & $20.61(24.87)$ & $10.51(14.19)$ & $16.83(15.72)$ & $19.52(24.88)$ \\
\hline
\end{tabular}

Notes: Times 1, 2, and 3 refer to the mood scales given prior to training, immediately afterward, or after the 2 nd anagram task, respectively

mood from time 2 to time 3 (pre and post the anagram task) was interpreted to measure emotional vulnerability.

For mood during the cognitive phase a mixed ANOVA was performed with Condition (benign vs. negative) and Presentation (written vs. spoken) as the between subject factors and Time (time 1 vs. time 2) as the within-subject factor. No significant main effect or interaction was observed $(F \mathrm{~s}<2)$. For mood during the stress phase all main effects and interactions were non-significant $(F \mathrm{~s}<1$ ), apart from a Time (time 2 vs. time 3$) \times$ Condition (benign vs. negative) $\times$ Presentation (written vs. spoken) interaction, which was marginally significant $F(1,90)=3.81, p=.05$, partial $\eta^{2}=.04$. Within the benign condition, there was no main effect of Time $(F<1)$ or Presentation, $F(1,51)=2.19, p=.1$, but there was a trend-level Presentation $\mathrm{x}$ Time interaction, $F(1,51)=3.22, p=.08$, partial $\eta^{2}=.06$. While there was no significant difference in mood between the written and spoken groups prior to completing the stressful anagram task, $t<1$, after completing the anagram task there was a trend for those in the spoken group to rate their mood as less negative than those in the written group, $t(51)=$ $1.81, p=.08$. In the negative condition, neither the main effects of Time or Presentation were significant, $F \mathrm{~s}<1$, and nor was the Time $\mathrm{x}$ Presentation interaction, $F(1,39)=1.14$, $p=.29$, suggesting no change in mood over the stressful anagram task in the negatively trained group (see Table 2).

\section{Discussion}

The present study is the first to provide preliminary evidence suggesting that the effects of the interpretation training procedure in children are differentiated according to the mode of 
presentation of the training material. Specifically, it was found that the spoken presentation of negative training material was more effective in training negative interpretation than written presentation of the same material, and in fact written presentation of the negative training materials had paradoxical effects, appearing to reduce the number of negative interpretations and perceived impact of negative social events from pre- to posttraining. While the benign training was broadly effective, there was less clear evidence for a differential effect of spoken or written presentation of the materials. These results may have interesting implications for our understanding of both the acquisition and remediation of negative cognitive biases in children. These areas will be discussed in turn with reference, first, to the negative training condition and, then, the positive training condition. The discussion will focus on understanding the differences between spoken and written presentation within the benign and negative conditions separately as this offers the most relevant and clearest interpretation of the study.

Investigating the experimental induction of negative cognitive biases in children can help develop our understanding of the acquisition of such biases in childhood and their role in the development of childhood anxiety. It is therefore interesting to have found differential effects of being required to either listen to the negative training material being spoken aloud by the experimenter or to read the same training material presented in written form. Children who heard the training material spoken aloud (spoken presentation) made more negative interpretations of ambiguous social events, and solved fewer difficult anagrams posttraining compared to pretraining. Conversely, while children who read the training material (written presentation) showed a similar decline in their performance on the anagram, otherwise they made fewer negative interpretations, and rated negative social events as having less emotional consequence.

This asymmetry in the superior efficacy of spoken over written presentation stands in contrast with the results reported by Mackintosh et al. (2006, Experiment 2) and Standage et al. (2009), who found that the presentation modality did not affect the magnitude of change in interpretation bias. However, these contrasting results can be reconciled if we consider that individuals from two different populations participated in these studies (adults vs. children). Another factor that may enhance the effectiveness of our training is that children listened to the stories and the feedback spoken out by the experimenter instead of through headphones as in the Standage et al. (2009). Thus, by involving an individual (i.e. the experimenter in this case) in bias modification training, the procedure followed in the current study may not only more closely mimic natural processes by which children come to learn particular interpretation styles (increasing the ecological validity of the experiment), but could even reflect a more powerful strategy in altering the interpretational styles than learning through computerized interface.

Notably, the results are in line with the conception that bias modification tasks closely mimic naturally occurring events. Describing their conception of environmental transmission, Field and Lester (2010) argue that:

The way in which this transmission of cognitive biases between parent and children might occur could be thought of rather like the bias modification tasks described previously. Anxious parents may transmit anxiety-related cognitive biases to their children through the provision of cues to threat and feedback on their cognitions and behaviours that act in a manner similar to cognitive bias modification. (Field and Lester, 2010, p. 260). 
This could be through verbal information (Field, Lawson and Banerjee, 2008) transmitted mainly via acoustic events, hence the superiority of the spoken presentation modality in inducing a negative interpretation bias. Therefore, the present data provide indirect and preliminary support for the notion that the cognitive bias modification procedures share similarities with a child's learning experiences with family members and/or other important individuals in their lives. Future research should seek to empirically test the notion that when children face ambiguity or potential threat in the presence of external agents (parents and siblings), and these agents do disambiguate the situation in a more negative way, these children tend to adopt a negative interpretative style. Furthermore, it is interesting to examine whether subtle, nonverbal cues and gestures on the part of external agents are equally or more effective in inducing a negative interpretative style compared to verbal cues and information, and whether the way in which the verbal information is delivered (e.g. non-emotional voice as in this study vs. a more emotionally-congruent delivery) affects the acquisition of an interpretive bias.

Turning to the positive condition, investigations of the factors that may modulate the beneficial effects of a paradigm designed to instil more positive cognitive biases in children are important for the development of effective novel interventions for the early intervention in or prevention of anxiety disorders. The benign training employed in the current study was broadly effective in that children receiving the benign training made significantly fewer negative interpretations of ambiguous events, more benign interpretations, and showed a trend to rate negative events as having less emotional impact from pre- to posttraining. These effects were the same whether the children listened to or read the benign training materials. The only task in which the mode of presentation of the training materials had a differential impact was on the difficult anagram task. Here the spoken presentation appeared to have been more beneficial, as children in the spoken presentation group solved significantly more anagrams and rated their mood as more positive than children in the written presentation group posttraining. Thus, spoken presentation may also be preferred for inducing positive training effects in children, but this may not be crucial.

There are a number of interesting and novel findings in the current study, one of which is the effect of the training on the behavioural measure used, the difficult anagram task. It is interesting that the induction of a negative interpretation bias affected participants' actual performance (number of anagrams solved). Thus, for the negatively trained group, anagram performance deteriorated from before to after the interpretation training, whereas for the benign group the performance remained stable. These results support the hypothesis that the negative interpretation training can negatively affect participants' performance, whilst undergoing a stressful experience, relative to benign interpretation training. As such this result may demonstrate one potentially important mechanism by which the detrimental effects of induced negative biases are translated into real-life, objective performance. We speculate that an important mediator of such effects would be children's sense of self-efficacy (Bandura, 1986; see also Lau et al., 2011; Dandeneau, Baldwin, Baccus, Sakellaropoulo and Pruessner, 2007), and this is an interesting avenue for future research. It is also interesting that the apparent changes in actual performance on the anagram task were not matched by changes in subjective performance. It is worth noting that similar discrepancies were found in the study by Lester et al. (2011), from which this anagram test was taken, and that factors that may contribute to a mismatch between actual and subjective performance may be useful to investigate further. 
Changing interpretation biases did not appear to invoke congruent changes in state negative mood from before the training to immediately afterwards. While this result is inconsistent with earlier work on interpretation training in adults (e.g. Mathews and Mackintosh, 2000), other work with adults and youth has also failed to observe significant results concerning the training-congruent effects on state anxiety (e.g. Salemink and Wiers, 2011; Salemink, van den Hout and Kindt, 2007). An advantage of the lack of direct effect of training on state mood is that it means that the differences observed in tasks carried out immediately posttraining are unlikely to be due to differences in induced mood between the experimental groups. However, it should be noted that for the measurement of negative mood, only three brief visual analogue scales were used as general measures of current feelings and their content did not quite match the content of the training.

One unexpected finding warrants further attention. Participants in the written presentation group trained to make negative interpretations actually interpreted ambiguous events less negatively and anticipated less negative consequences of these events compared with their baseline reports. It is difficult to account for this unexpected finding, but it could possibly be due to the fact that participants in the written group can progress through the experiment at their own individual pace and do not process them according to a pre-set rate that is inconsistent with their own natural speed. It has been suggested that most healthy people are characterized by a mildly positive bias that acts as a protecting factor from stress (e.g. Hirsch and Mathews, 2000). Given that only healthy preadolescents with moderate to high levels of social anxiety participated in this study, it is reasonable to assume that participants in the written group may have had more time to compare their own spontaneous (benign) responses with those presented to them as "correct" and thus to refute or dismiss the negative explanations, with the resulting outcome of sticking to their initial perception of the situation. In contrast, for participants presented with benign interpretations in written form, the "correct" feedback may not have clashed with their spontaneous (positive) responses, thus avoiding evaluation and dismissal of the explanations, and allowing the training to influence their biases in the intended direction. These results are consistent with findings from the adult literature that encouraging a verbal, comparative mode of processing of training material can reduce or even reverse its impact (Holmes et al., 2009).

Given that prolonged cognitive bias modification tasks are often experienced as boring or monotonous by participants (Brosan, Hoppitt, Shelfer, Sillence and Mackintosh, 2011), it is encouraging that this short, scenario-based interpretation training was successful in inducing a positive interpretation and judgmental bias. Overall, the benignly trained group endorsed fewer negative interpretations and more benign interpretations and tended to report less negative emotional consequences compared with their endorsements at baseline, regardless of mode of presentation. Therefore our study has not only replicated previous findings (Vassilopoulos et al., 2009, 2012) but also extended them by demonstrating that the effects of benign training on interpretation bias can be induced with a minimal training consisting of only 15 brief social scenarios presented either visually or aurally in a sample of primary school children; this therefore corroborates similar positive results from a recent study (Vassilopoulos and Moberly, in press). Although the differential effects of spoken or written presentation of training scenarios were not pronounced for the benign training, as the spoken training took less time to administer and could be administered with minimal resources to several children simultaneously, regardless of their reading ability, it may have practical advantages as a lowintensity clinical intervention. Nevertheless, it is not clear whether or how the current results 
could be influenced by demand effects, and future studies should seek to employ automatic and objective tasks to investigate the induction of interpretation biases (e.g. lexical decision paradigm). Further, the long term effects of the brief interpretation training approach used in this study are not yet clear.

As in all studies, this work has a number of limitations beyond those already mentioned. Most obviously, although children scoring above the mean score on a social anxiety scale were included, they were still scoring within the normal range for this scale and thus the present results cannot be generalized to clinical samples. Additionally, it is important in terms of clinical utility for future studies to demonstrate an influence of interpretation training effects on emotional vulnerability, across a wide range of measures including physiological and behavioural (such as a social challenge task) in addition to the more common self-report measures. Another limitation of the present study is that the rate of stimuli presentation was not matched for the two presentation modes so it is not possible to arrive at definite conclusions about the superiority of one condition over the other. Following Standage et al. (2009), the instructions for the VAS mood scales matched the presentation mode of the training (i.e. spoken for the spoken presentation group and written for the written presentation group). However, this makes it more difficult to be certain about comparisons between the written and spoken presentation conditions on this particular measure. Further, the absence of a "no-contingency" control group makes it difficult to rule out the possibility that some of the results are the effect of repeated exposure to the training material and the testing situation.

In conclusion, we found that the written and spoken presentation of the training material differentially affected training-congruent change in interpretation bias and judgments of negative consequences in children and that this translated into associated change in performance during a difficult anagram task. These results not only shed light on the mechanism of the genesis of interpretation bias in children, but also highlight the need for future studies to systematically investigate the impact of instructions and presentation mode on the effectiveness of interpretation interventions, for the development of effective treatment packages for childhood anxiety disorders.

\section{Acknowledgments}

This study was supported by the "K. Karatheodoris" research grant (contract C919) awarded to the first author by the Research Committee of the University of Patras. The second author was supported by the Lupina Foundation and the Medical Research Council (MC-A0605PR50). The funders had no involvement in the study design, data collection, analysis, interpretation or writing of the paper.

\section{References}

Bandura, A. (1986). Social Foundations of Thought and Action. New Jersey: Prentice Hall.

Beard, C. (2011). Cognitive bias modification for anxiety: current evidence and future directions. Expert Review of Neurotherapeutics, 2, 299-311.

Beard, C. and Amir, N. (2008). A multi-session interpretation modification program: changes in interpretation and social anxiety symptoms. Behaviour Research and Therapy, 46, 1135-1141.

Brosan, L., Hoppitt, L., Shelfer, L., Sillence, A. and Mackintosh, B. (2011). Cognitive bias modification for attention and interpretation reduces trait and state anxiety in anxious patients referred 
to an out-patient service: results from a pilot study. Journal of Behavior Therapy and Experimental Psychiatry, 42, 258-264.

Dandeneau, S. D., Baldwin, M. W., Baccus, J. R., Sakellaropoulo, M. and Pruessner, J. C. (2007). Cutting stress off at the pass: reducing vigilance and responsiveness to social threat by manipulating attention. Journal of Personality and Social Psychology, 93, 651-666.

Field, A. P. (2006a). The behavioral inhibition system and the verbal information pathway to children's fears. Journal of Abnormal Psychology, 115, 742-752.

Field, A. P. (2006b). Watch out for the beast: fear information and attentional bias in children. Journal of Clinical Child and Adolescent Psychology, 35, 431-439.

Field, A. P., Lawson, J. and Banerjee, R. (2008). The verbal threat information pathway to fear in children: the longitudinal effects on fear cognitions and the immediate effects on avoidance behavior. Journal of Abnormal Psychology, 117, 214-224.

Field, A. P. and Lester, K. J. (2010). Learning of information processing biases in anxious children and adolescents. In J. A. Hadwin and A. P. Field (Eds.), Information Processing Biases and Anxiety: a developmental perspective (pp. 253-279). Chichester: Wiley.

Greene, R. L., Elliott, C. L. and Smith, M. D. (1988). When do interleaved suffixes improve recall? Journal of Memory and Language, 27, 560-571.

Hirsch, C. R. and Mathews, A. (2000). Impaired positive inferential bias in social phobia. Journal of Abnormal Psychology, 109, 705-712.

Holmes, E. A., Lang, T. J. and Shah, D. M. (2009). Developing interpretation bias modification as a "cognitive vaccine" for depressed mood: imagining positive events makes you feel better than thinking about them verbally. Journal of Abnormal Psychology, 118, 76-88.

Holmes, E. A. and Mathews, A. (2005). Mental imagery and emotion: a special relationship? Emotion, 5, 489-497.

Holmes, E. A., Mathews, A., Dalgleish, T. and Mackintosh, B. (2006). Positive interpretation training: effects of mental imagery versus verbal training on positive mood. Behavior Therapy, 37, 237247.

Kovacs, M. K. (1992). Children's Depression Inventory-Short Form (CDI). New York: Multi-Health Systems Inc.

La Greca, A. M. and Stone, W. L. (1993). The Social Anxiety Scale for Children-Revised: factor structure and concurrent validity. Journal of Clinical Child Psychology, 22, 17-27.

Lau, J. Y., Molyneaux, E., Telman, M. D. and Belli, S. (2011). The plasticity of adolescent cognitions: data from a novel cognitive bias modification training task. Child Psychiatry and Human Development, 42, 679-693.

Lester, K. J., Field, A. P. and Muris, P. (2011a). Experimental modification of interpretation bias regarding social and animal fear in children. Journal of Anxiety Disorders, 25, 697-705.

Lester, K. J., Field, A. P. and Muris, P. (2011b). Experimental modification of interpretation bias about animal fear in your children: effects on cognition, avoidance behaviour, anxiety vulnerability and physiological responding. Journal of Clinical Child and Adolescent Psychology, 40, 864-877.

Lester, K. J., Mathews, A., Davison, P. S., Burgess, J. L. and Yiend, J. (2011). Modifying cognitive errors promotes cognitive well being: a new approach to bias modification. Journal of Behavior Therapy and Experimental Psychiatry, 42, 298-308.

Lothman, C., Holmes, E. A., Chan, S. W. and Lau, J. Y. (2011). Cognitive bias training modification in adolescents: effects on interpretation biases and mood. Journal of Child Psychology and Psychiatry, $52,24-32$.

Mackintosh, B., Mathews, A., Yiend, J., Ridgeway, V. and Cook, E. (2006). Induced biases in emotional interpretation influence stress vulnerability and endure despite change in context. Behavior Therapy, 37, 209-222.

MacLeod, C. and Mathews, A. (2012). Cognitive bias modification approaches to anxiety. Annual Review of Clinical Psychology, 8, 189-217. 
MacLeod, C., Rutherford, E., Campbell, L., Ebsworthy, C. and Holker, L. (2002). Selective attention and emotional vulnerability: assessing the causal basis of their association through the experimental manipulation of attentional bias. Journal of Abnormal Psychology, 111, 107-123.

Mathews, A. and Mackintosh, B. (2000). Induced emotional interpretation bias and anxiety. Journal of Abnormal Psychology, 109, 602-615.

Miers, A. C., Blöte, A. W., de Rooij, M., Bokhorst, C. L. and Westenberg, P. M. (in press). Trajectories of social anxiety during adolescence and relations with cognition, social competence, and temperament. Journal of Abnormal Child Psychology.

Miers, A. C., Blöte, A. W. and Westenberg, P. M. (2011). Negative social cognitions in socially anxious youth: distorted reality or a kernel of truth? Journal of Child and Family Studies, 20, 214223.

Muris, P. (2010). Anxiety-related reasoning biases in children and adolescents. In J. Hadwin and A. Field (Eds.), Information-Processing Biases and Anxiety: a developmental perspective (pp. 21-46). Chichester, UK: Wiley.

Muris, P. and Field, A. P. (2008). Distorted cognition and pathological anxiety in children and adolescents. Cognition and Emotion, 22, 395-421.

Muris, P. and Field, A. P. (2010). The role of verbal threat information in the development of childhood fear. 'Beware the Jabberwock!'. Clinical Child and Family Psychology Review, 13, 129-150.

Muris, P., Huijding, J., Mayer, B. and Hameetman, M. (2008). A space odyssey: experimental manipulation of threat perception and anxiety-related interpretation bias in children. Child Psychiatry and Human Development, 39, 469-480.

Muris, P., Huijding, J., Mayer, B., Remmerswaal, D. and Vreden, S. (2009). Ground control to Major Tom: experimental manipulation of anxiety-related interpretation bias by means of the "space odyssey" paradigm and the effects on avoidance tendencies in children. Journal of Anxiety Disorders, 23, 333-340.

Muris, P., Huijding, J., Mayer, B., van As, W. and van Alem, S. (2011). Reduction of verbally learned fear in children: a comparison between positive information, imagery, and a control condition. Journal of Behavior Therapy and Experimental Psychiatry, 42, 139-144.

Muris, P., Rassin, E., Smeets, G., Mayer, B., Huijding, J., Remmerswaal, D., et al. (2009). Effects of verbal information on fear-related reasoning biases in children. Behaviour Research and Therapy, 47, 206-214.

Murphy, R., Hirsch, C. R., Mathews, A., Smith, K. and Clark, D. M. (2007). Facilitating a benign interpretation bias in a high socially anxious population. Behaviour Research and Therapy, 45, 15171529.

Muthén, B. and Kaplan, D. (1992). A comparison for some methodologies for the factor analysis of non-normal Likert variables: a note on the size of the model. British Journal of Mathematical and Statistical Psychology, 45, 19-30.

Rachman, S. (1977). The conditioning theory of fear acquisition: a critical examination. Behaviour Research and Therapy, 15, 375-387.

Rapee, R. M. and Spence, S. H. (2004). The etiology of social phobia: empirical evidence and an initial model. Clinical Psychology Review, 24, 737-767.

Salemink, E., van den Hout, M. and Kindt, M. (2007). Trained interpretive bias and anxiety. Behaviour Research and Therapy, 45, 329-340.

Salemink, E. and Wiers, R. W. (2011). Modifying threat-related interpretive bias in adolescents. Journal of Abnormal Child Psychology, 39, 967-976.

Sloutsky, V. M. and Napolitano, A. C. (2003). Is a picture worth a thousand words? Preference for auditory modality in young children. Child Development, 74, 822-833.

Standage, H., Ashwin, C. and Fox, E. (2009). Comparing visual and auditory presentation for the modification of interpretation bias. Journal of Behavior Therapy and Experimental Psychiatry, 40, $558-570$. 
Vassilopoulos, S. and Banerjee, R. (2008). Interpretations and judgments for positive and negative social scenarios in childhood social anxiety. Behaviour Research and Therapy, 46, 870-876.

Vassilopoulos, S., Banerjee, R. and Prantzalou, C. (2009). Experimental modification of interpretation bias in socially anxious children: changes in interpretation, anticipated interpersonal anxiety, and social anxiety symptoms. Behaviour Research and Therapy, 47, 1085-1089.

Vassilopoulos, S. P., Blackwell, S. E., Moberly, N. J. and Karahaliou, E. (2012). Comparing imagery and verbal instructions for the experimental modification of interpretation and judgmental bias in children. Journal of Behavior Therapy and Experimental Psychiatry, 43, 594-601.

Vassilopoulos, S. P. and Moberly, N. J. (in press). Cognitive bias modification in pre-adolescent children: inducing an interpretation bias affects self-imagery. Cognitive Therapy and Research. 